\title{
Electronic research: An opportunity for the Society for Neuro-Oncology
}

\author{
Eric. D. Kramer and Oliver Bögler ${ }^{1}$ \\ Cooper Health System/Robert Wood Johnson Medical School at Camden, Camden, NJ 08103 (E.D.K.); and The \\ Hermelin Brain Tumor Center, Department of Neurosurgery, Henry Ford Hospital, Detroit MI 48202 (O.B.)
}

S $\mathrm{NO}^{2}$ acts as a unifying body lending identity and focus to the neuro-oncology community. It achieves this by providing a structure for organization and a conduit for communication through its annual meeting and its journal, Neuro-Oncology. Although the meeting provides a forum, it occurs but once a year; and, although the journal effectively disseminates information, it does so in only one direction. New modes of communication are now available that, we argue, have the added potential of not only disseminating existing information but also actually generating new findings and insights. We contend that because SNO has had its inception during, and perhaps in some measure because of, the revolutionary information age, a logical extension of the Society's mission would be to facilitate the advancement of e-res through the exploitation of electronic communications. The pursuit of our commonly stated goal to find new and better approaches to diagnose and treat brain cancer compels us to break out of the culture of the single lab and, primarily, single-institution experience. We cannot afford to continue doing business as usual while medical science as a whole is experiencing exponential growth and electronic devices are changing the way in which we live. Our patients, neuro-oncology research, and every individual investigator could benefit if SNO were to position itself as a nidus from within which the field of e-res could be developed and maximized.

In the fall of 1999, a short epidemiologic survey regarding adult primary neuroectodermal tumor was

Received 5 December 2000, accepted 12 February 2001.

\footnotetext{
${ }^{1}$ Address correspondence and reprint requests to Oliver Bögler, The Hermelin Brain Tumor Center, Depart. of Neurosurgery, Henry Ford Hospital, 2799 W. Grand Blvd., Detroit MI 48202.
}

${ }^{2} \mathrm{Abbreviations}$ used are as follows: e-res, electronic research; SNO, Society for Neuro-Oncology. offered through the SNO Web site (www.soc-neuroonc.org). The site appeared to be an optimal interface at which the collective experience of neuro-oncologists could be reviewed, epidemiologic observations gathered, and the results disseminated. SNO member participation could be increased by the sharing of data between large institutions and smaller practices. This exercise was structured to provide both a meaningful contribution to the understanding of an interesting topic and some insight into how SNO might exploit the Internet beyond the current use of providing annual meeting abstract submission and information, meeting calendars, job and grant boards, and a member directory. Unfortunately, but not wholly unexpectedly, the response to the primary neuroectodermal tumor questionnaire was disappointing. Comments were made concerning the questions asked and the construction of the tool, but few offered their data.

A similar experience was registered regarding a discussion forum that remains present, but inactive, at SNO's Web site. In response to a communiqué by thenpresident Dr. Mitch Berger, a voluntary, password-protected, member-only message board area was created. It was designed to replace the sporadic discussions on specific cases that erupt from time to time on SNO's e-mail list, which was intended for the dissemination of important SNO business only. However, it was not possible to get a critical mass of people to sign up, and no discussions have taken place at this Web address.

These two failed overtures can be looked at as opportunities. A series of essentially self-evident questions are raised by these experiences, and their discussion may lead to insights on how e-res can be pursued successful. How the broad membership of SNO responds to the challenges and opportunities presented by the availability of e-res will impact the perception of SNO and our operations far into the future. The purpose of this editorial is to stimulate a discussion by the membership about e-res. Our aim is to arrive at acceptable definitions and begin a dialogue 
with the goal of building a consensus that may direct future endeavors.

\section{E-res: A definition}

Before a meaningful discussion on e-res can be convened, a definition is needed, and we would like to offer the following. E-res is the gathering, examination, and dissemination of data using electronic means. This intentionally broad definition would include a message board on clinical cases soliciting input from colleagues and would provide a platform for epidemiological studies. We have intentionally chosen to include research in this term because of its own broad definition to cover anything from a case report to bench work. The choice of this term does not imply that all activity conducted relative to e-res will be research conducted for the intent of publication, but will also include other academic and scientific communication. Alternate terms were considered and concluded to be either too restrictive or too nondescript. Collection of clinical data from multiple centers and a gene expression profiling study from multiple laboratories are examples of the types of studies that could be accomplished. The common element is that of crossing organizational boundaries and using electronic media to enable "real-time" and ongoing progress.

\section{Can e-res improve what we are already doing and allow us to do new things?}

The significance of this discussion is predicated on whether e-res can improve how things are currently done, or, indeed, whether it facilitates anything new. Here we discuss some examples that are also outlined in the Table. The main improvements that e-res offers would be derived by taking advantage of the inherent capabilities of the Internet. These include universal access (among physicians and scientists in North America, SNO's geographical mandate) and low cost and rapid communications.
Because ideas and information can be discussed and shared on a continuous basis, this creates the opportunity to have widespread and timely input for concept evaluation, study development optimization, and rapid and accessible data analysis. The sustained nature of such an exchange, and the option to participate at one's convenience, suggests that e-res can support discussions in a new way that would supplement and support the annual meeting. At the simplest level, such discussion could be exclusively text-based in the familiar message-board format. However, a Web site such as www.biocarta.com, which allows participants to submit graphics of molecular pathways that are linked to gene databases and that can be commented on by visitors, suggests that this type of site may become yet more useful and active. This will be primarily achieved by expanding on the other strengths inherent in the Internet: the ease of collecting visitor input, the deployment of multimedia content that is richer than a printed page and more permanent than a seminar, and the ability to conveniently link data sources from disease descriptions to gene sequences.

A concrete example of how e-res of this type could significantly improve how we currently do things would be an online site dedicated to a particular topic of wide interest to researchers. For example, the recent explosion of animal models for brain tumor research has made keeping up to date with the relative characteristics of these models challenging for someone not intimately familiar with this specialty. Such a site could include a catalog of descriptions of models in terms of genetic origins, histology, tumor progression, gene expression, and model uses. Links to published papers and relevant genes would be included. Each model would be associated with a discussion area where interested researchers could interface with the inventors and users of a particular model. The contents of this site would need to be curated and the discussion moderated by an individual or group that would function much like the authors of a review article. The significant advantage that such a site would have over an article that might become rapidly outdated is its dynamic and interactive aspects. Furthermore,

Table. Potential uses of e-res

Activity

Communication

Clinical trials

Gene expression studies

Brain tumor animal models
Problems or limitations

Limited communication among SNO members except for annual meeting

Difficult and slow accrual of patients for rarer histologies

Time-consuming methods for sharing data of ongoing clinical trials

Voluminous data on gene expression

Lagging tools for compiling and analyzing data

Competing models from which to choose, each with its own strengths and weaknesses

Voluminous and not-well-collated data for some models (e.g., cell lines)
General discussion and exchange

Topic-specific sites

Project development: concept interest, concept

refinement, and collaborator identification

Ongoing peer review

Internet data collection and exchange on specific topics

Online database of cell lines, tumor types, and expressed genes

Online compendium of models with a searchable interface to facilitate selection

"How to" information

Online forum for establishing collaborations 
advances could be rapidly disseminated via e-mail on a subscription basis.

Beyond the realm of simple information exchange, e-res provides the opportunity to coordinate collaborative research endeavors at every stage. From recruiting interested individuals to allowing widespread and timely input for concept evaluation and study design optimization, from data collection and analysis to coordination of publication, e-res can facilitate research. The sustained nature of such an exchange, and the option to participate at one's convenience, suggests that e-res can support discussions in a new way that would supplement and support the SNO annual meeting. Indeed, in a small way, the writing of this editorial exemplifies the stages that such a project might take:

- An idea/concept was addressed to a fellow member of SNO

- Interest was gauged

- A collaborator was obtained

- The concept was refined by repeated electronic conversation

- Action ensued toward a completed project

- Progress was tracked

- The completed project was submitted for feedback and ultimately published.

This process is, of course, not unique and might be considered a summary of essentially every endeavor. The difference here is that these authors have worked on this project entirely by computer through the SNO site, never having met in person.

E-res can also do things that are currently not done. In general terms, tools that collect information from participants expeditiously and cost-effectively can be readily deployed, and so e-res makes the routine collation of data from multiple centers possible. Centralized data could, in turn, be made available to all appropriate investigators, and information could flow essentially seamlessly and nearly freely, accruing not only savings in money but also in time. Quality would need to be maintained and preserved by having multiple de facto reviewers participate, and their involvement could be likened to the process associated with writing review articles or textbooks. This mechanism of data collection and sharing is increasingly being used for multicenter clinical trials, and it represents the closest example at hand. However, we contend that extending this approach to ad hoc or project-specific groups could be of even more value. Indeed, groups such as SNO could play a key role in facilitating e-res for groups that do not have associated data management and information technology personnel, such as are found in clinical trials consortia.

The advent of e-res appears to offer a new way to perform some studies. Specifically, pure data collection studies, such as epidemiological reviews, probably could be accomplished expeditiously through this approach. However, e-res offers more than just data collection. Here is an opportunity to accelerate the time-honored process of building consensus in both the fields of clinical and basic research to identify new findings and technologies. The technical and procedural specifics will need to be tailored to each particular undertaking, but limitations are set only by our collective initiative and creativity. For example, comparisons of the clinical course of a specific tumor type at multiple centers could be made, which might more rapidly reveal successful approaches. Another example comes from a currently topical area: the use of biochip arrays to analyze gene expression. In this field, the initial phase of enthusiasm typical of the advent of a new and very powerful technology is gradually giving way to the realization that the rapid data cascade needs to be tempered by mechanisms to ensure cross-platform and cross-center consistency. The very nature and volume of the data generated by the new technologies that are transforming biology and medicine require the use of computers and so suggests e-res. Databases, accessed through Web sites, could allow all interested parties to enter, for example, relative expression levels of genes in different glioma subtypes in an attempt to reach a consensus and so work toward a standard. The timeliness of this is demonstrated by the recent launch of the Gene Expression Omnibus (http://www.ncbi.nlm.nih.gov/geo/) by the National Center for Bio-Informatics. This site allows researchers who have data from gene expression arrays to upload the information to a public database that all can access. A case might be made for a smaller database focused on the area of neuro-oncology.

\section{What are the barriers to e-res?}

Physician resistance to e-res is real and must be addressed. On one level, e-res suffers from the same barriers to adoption as any new technology or mode of pursuing existing goals. To overcome these barriers, it is necessary to ensure that the long-term benefits outweigh the short-term inconvenience associated with the steep end of the learning curve. The benefits that individuals derive from participation will be project specific, and also to an extent, individual specific, so only general themes can be discussed here.

We expect that physicians and scientists who deal with high-level technology on a routine basis would not perceive the technological nature as a barrier in itself. However, these individuals are acutely subject to severe time constraints, which makes taking on any new endeavor a difficult decision. This is where the ability to interact with e-res projects on one's own schedule becomes an important factor. Active participants are likely to garner more from e-res projects, just as regular seminar attendees gain more by their participation. Unlike a seminar, however, which is a transient event, e-res offers a more permanent source of information and so can also be very valuable to the more sporadic visitor. In addition, the arguments that the "old way has worked pretty well" and "no one else is doing it" are likely to be raised by some, as is the question of "what's in it for me?" We contend that in a welldesigned e-res project it will become clear that the new way offers significant advantages that transcend the fact that no one else is doing it and clearly show what is in it for all participants. The ultimate measure will be whether a given e-res project accelerates real advances in understanding. Projects that don't, will not survive.

E-res must also overcome the barrier of integration with traditional measures of productivity, primarily pub- 
lication of peer-reviewed manuscripts. The issue of potential compromise of career advancement by sharing authorship on papers that would result from broad-based e-res is real. Institutions or individuals may simply not see the gain from enfranchising potentially competing institutions, organizations, labs, or groups. In certain areas of research, however, large groups are more effective and make more rapid progress than do individuals. The various genome projects are obvious examples. Note also that electronic data sharing is now the norm in this field. Increased productivity could result in an individual actually producing more papers, with more authors per paper. Over the coming decade, e-res may gain sufficient acceptance to be recognized as a form of publication in itself. Electronic publishing is gaining acceptance, and now many print journals also publish on the Web, including Neuro-Oncology. Several journals prepublish electronically immediately upon acceptance, for example The Journal of Biological Chemistry. Others, for example Breast Cancer Research, are publishing the full versions of papers online, with synopses in the paper edition. Whether an e-res project itself may eventually be considered equivalent to publication remains an open question. To return to one of the examples used above, do the curators of a Web site concerning brain tumor animal models benefit as much from this activity as they would by writing a review on the subject every 5 years? We would argue that any loss of a publication would be made up by the increase in meaningful collaborations and shared information. By embracing e-res, $\mathrm{SNO}$ will be perceived as a leader in this inexorable advancement.

An unavoidable "life-or-death" issue that must be addressed is that of data security and reliability. A method of delimiting or controlling participation would clearly be necessary. Nevertheless, the sharing of data across public elements of the Internet adds risks. Publicly accessible data repositories are also vulnerable to corruption for that reason. We believe that the significance of this problem should not be underestimated, and furthermore, with the ongoing arms race between hackers and computer security experts, it is not likely to go away. The best approach would be to assume that any shared data repository is vulnerable and so build in the necessary safeguards at the lowest level. In the case of clinical projects, this could include coding the personal identification information of patients, as is becoming standard practice for any data that moves beyond the firewall of the treating institution.
In addition, e-res, by its collaborative nature, requires management of relationships between researchers and institutions. In this regard, it is no different from collaborations that, at present, spring up naturally between individual investigators. Simple letters of agreement would suffice for the simplest level of e-res, whereas longer-term projects involving more institutions would require more formal agreements.

\section{Should, or could, the SNO establish itself or its members as leaders in the use of e-res?}

Science and medical research are intricate parts of the machinery of human industry. In that sense, we argue that e-res is already here, and the question is not whether it will be adopted, but when. As SNO emerges as the de facto voice for adult neuro-oncology in North America, it faces the opportunity to facilitate this transition for its members and this field. At one level there is the opportunity to study the development of e-res in neuro-oncology and determine who will participate in what fashion and why. This information could be used to design strategies, including meetings and perhaps even incentives, that could further this avenue of interaction. A position statement issued by SNO officers or its board would be innovative, instructional, and directing. A good starting point might be to provide incentives to pioneers willing to take risks in this area. Perhaps a sponsor could be found who would be willing to provide funds for a small annual grant specifically for e-res in neuro-oncology, the awarding of which could be overseen by SNO. Another area that SNO leadership could investigate is whether it could host e-res collaboration through its own Web site.

In closing, the first issue offered for consideration by $\mathrm{SNO}$ membership is how to respond to e-res. Here is a chance to be leaders in the research community and pioneers in the e-res domain of cyberspace. Those who respond will essentially act as the development and advisory panel of this evolution. We invite all to participate in this discussion by logging on to www.soc-neuro-onc. org/forum.

Neuro-Oncology 3, 211-214, 2001 (Posted to NeuroOncology [serial online], Doc. 00-065, May 15, 2001. URL <neuro-oncology.mc.duke.edu>) 